\title{
Multiwavelength Monitoring of the Be/X-ray Binary X Persei - Evidence for Multiple Disk Structures
}

\author{
P. Roche
}

\author{
Dept. of Physics $\&$ Astronomy, University of Leicester, England
}

\author{
A.E. Tarasov
}

Crimean Astrophysical Observatory, Nauchny, Crimea, 334413 Ukraine

\author{
V.M. Lyuty
}

Sternberg Astronomical Institute, Universitetskii pr. 13, Moscow, Russia

\section{J.S. Clark}

Astronomy Center, University of Sussex, Brighton, England

\section{Larionov}

Astronomical Institute, St.Petersburg University, St.Petersburg, Russia

\begin{abstract}
We present optical photometry and high resolution optical spectra of the Be star X Persei/HD 24534, the counterpart to the Xray pulsar $4 U 0352+30$, obtained over the past $\sim 10$ years. These spectra show a variety of behaviour, ending with the onset of a strong $V / R$ cycle. Observations of the He I6678 line suggest several episodes of discrete disc forming. This data forms part of a major long-term multiwavelength monitoring programme ( $U B V R I J H K L$ photometry plus optical and UV spectroscopy).
\end{abstract}

\section{Introduction}

$\mathrm{X}$ Persei/HD 24534 is the prototypical persistent low luminosity Be/X-ray binary system. The Be star has recently been reclassified as a B0 Ve at a distance of around $700-800 \mathrm{pc}$. Recently the system has undergone two episodes of dramatic disk loss, during the mid-1970's and the 1980's, and following the latter event was seen to display unusual He I 6678 line profiles. Here we present optical data obtained over the period 1987 to 1998, covering the entire disk loss period and the unusual multi-component line behaviour.

Most of the data presented here was obtained from the Crimean Astrophysical Observatory, Ukraine, $2.6 \mathrm{~m}$ Shain telescope, Coude focus with CCD, resolution $\sim 25,000$. Photometry was obtained with the Sternberg Astronomical Institute $0.60 \mathrm{~m}$ and the Univ. of St. Petersburg $0.74 \mathrm{~m} \mathrm{IR}$ telescopes, both also located at Crimea, Ukraine. 

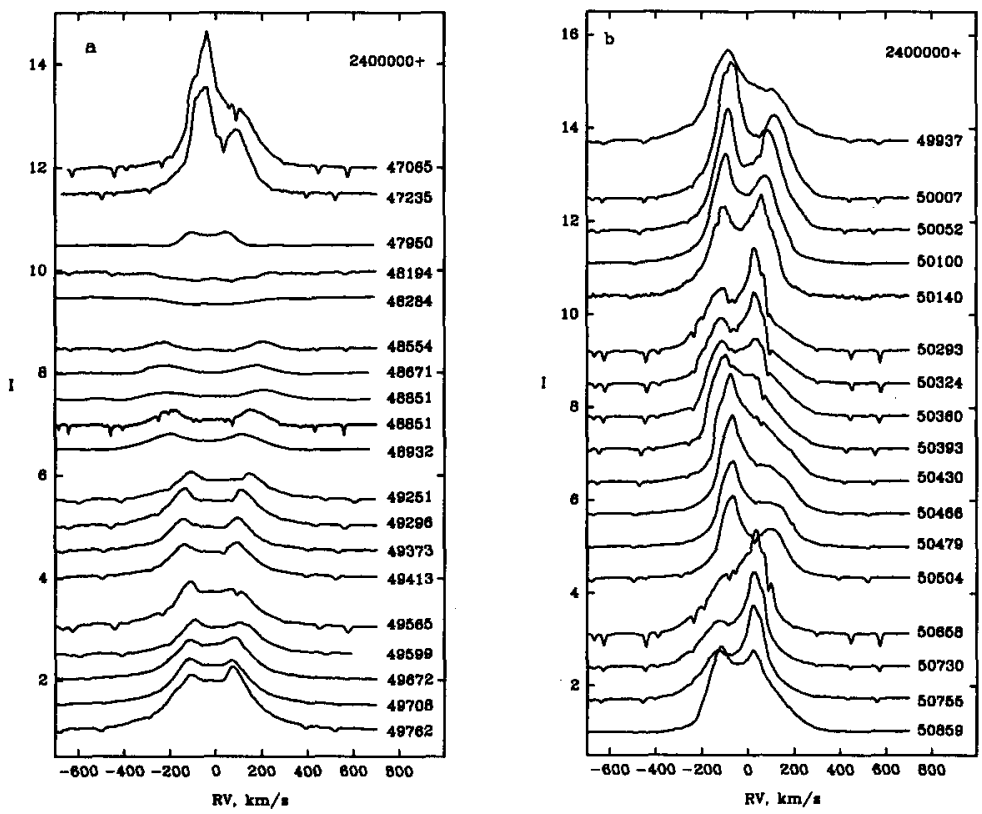

Figure 1. The H $\alpha$ spectra of X Per covering the period 1987-1998.

\section{Behaviour of the $\mathrm{H} \alpha$ and $\mathrm{He} I 6678$ lines.}

Figure 1, panels $a$ and $b$ show the $\mathrm{H} \alpha$ line of X Per. Figure 3 (left panel) displays the observed parameters of the $\mathrm{H} \alpha$ line taken from the spectra in Figure 1. Figure 2 panels $a$ and $b$ show the behaviour of the He I 6678 line. Figure 3 (right panel) displays the observed line parameters of the He I 6678 line from the spectra shown in Figure 2.

Phase 1 (TJD 46500-47700): covers bright state prior to Extended Low State (ELS), showing double-peaked structure (see Phase 3), followed by rapid fading to minimum brightness $\left(\Delta V \sim 0.6^{m}\right.$ in $\sim 400$ days $)$

$\mathrm{H} \alpha$ : Region is poorly covering spectroscopically, but our data shows that the $\mathrm{H} \alpha$ line is bright ( $\mathrm{EW} \sim 10 \AA$ ), with double-peaked structure $(\mathrm{V}>\mathrm{R}$ ). There may be some evidence for to suggest we are seeing possible $V / R$ variations. Small peak separation $\left(\sim 140 \mathrm{~km} \mathrm{~s}^{-1}\right)$.

(No He I 6678 data for this phase)

Phase 2 (TJD 47700-49100)): star in extended low state, with very low amplitude photometric variations, possible increasing in size towards end of this period.

$\mathrm{H} \alpha$ : Starts as weak, double-peaked emission line, falling to photospheric absorption line for a short period ( $\sim 20-30$ days). Rapid EW decrease, but lagging behind $V$ decrease by about 400-500 days. After absorption line seen, weak emission returns with large peak separation $\left(\sim 500 \mathrm{~km} \mathrm{~s}^{-1}\right)$. Intensity of the line gradually increases. As EW increases almost linearly, peak separation decreases. No $V / R$ variability observed. 

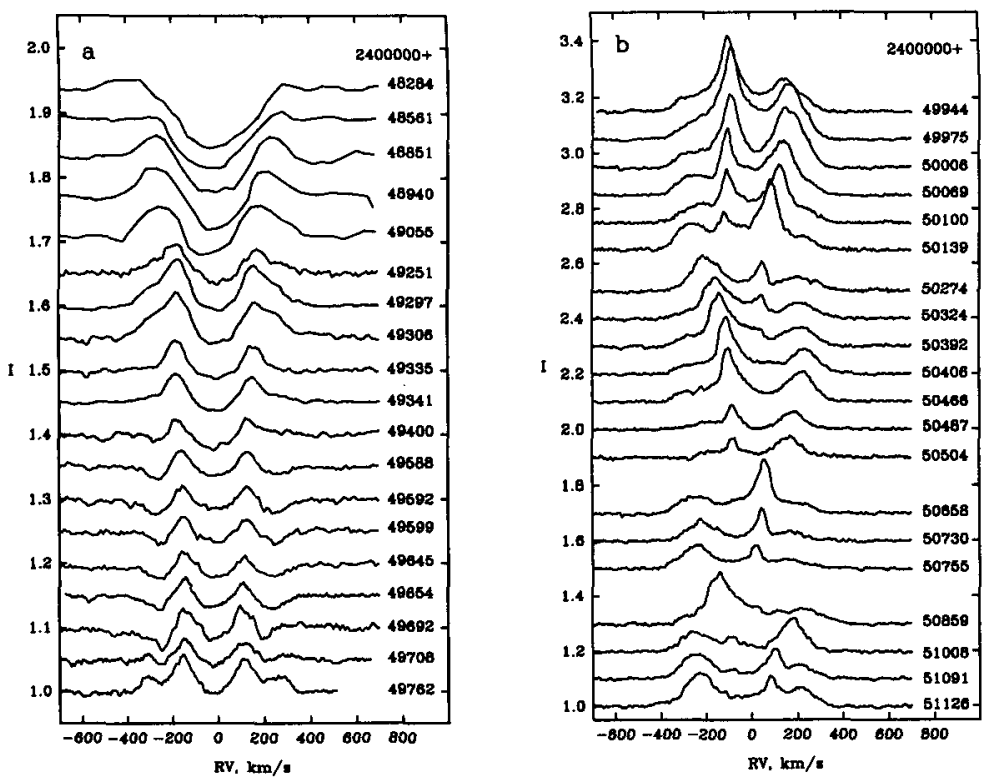

Figure 2. The He I6678 line spectra covering the period 1991-1998.

He I6678: First observation is in absorption, line gradually moves into emission with widely separated peaks and central absorption component.

Phase 3 (TJD 49100-49650): ELS ends with rapid brightening in $V\left(\Delta V \sim 0.6^{m}\right.$ in 550 days), again shoving double-peaked structure as for previous maximum (Phase 1).

$\mathrm{H} \alpha$ : EW reaches plateau (whilst $V$ still increasing), some low amplitude, irregular V/R variability, peaks continue to move closer at same rate as in Phase 2. He I6678: Peak intensity decreases, separation decreases, photospheric wings appear at edge of the emission peaks. EW variability more pronounced than for $\mathrm{H} \alpha$, reaching a local minimum when star is at maximum brightness (end of Phase 3).

Phase 4 (TJD 4965-5000): rapid fading in $V$ magnitude $\left(\Delta V \sim 0.55^{m}\right.$ in 350 days).

$\mathrm{H} \alpha$ : During this period, the $\mathrm{H} \alpha$ line profile shows large global changes in structure, remaining strong and double-peaked throughout. EW begins to increase again (anti-correlated with $V$ changes), and we see the onset of the long-term V/R cycle, which begins near maximum brightness (amplitude increasing with time). Peak separation decreases at much slower rate.

He I6678: Disappearance of photospheric wings, replaced by additional emission components at high velocity (four peak structure). EW behaviour as for $\mathrm{H} \alpha$ (increases while $V$ decreases), peak separation remains unchanged for main (inner) components.

Phase 5 (TJD 50000-50800): minimum of light curve at $V \sim 6.7^{m}$, slightly 

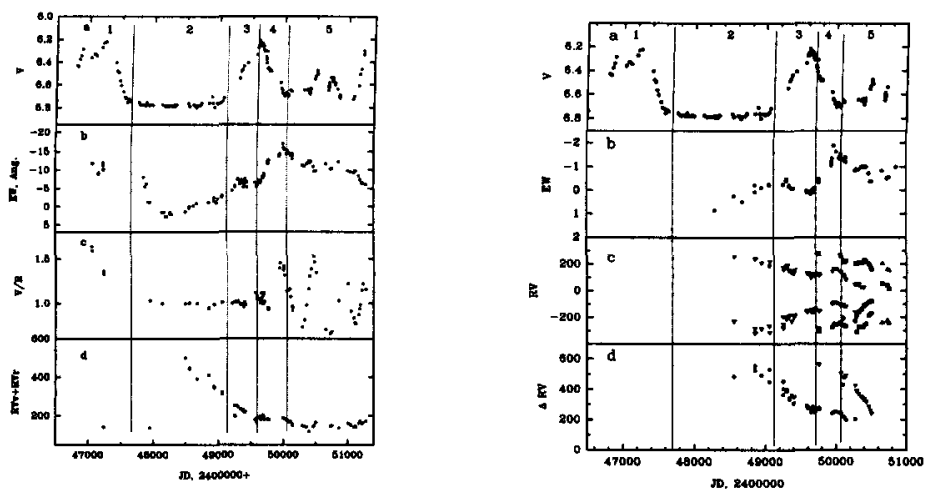

Figure 3. Time variability of the parameters of the $\mathrm{H} \alpha$ (left panel) and He I 6678 (right panel) lins.

brighter than in ELS ( $\left.V \sim 6.8^{m}\right)$ suggesting some overall long-term brightening. Some small brightening episodes $\left(\Delta V \sim 0.1-0.2^{m}\right)$, but poor coverage.

$\mathrm{H} \alpha$ : Pronounced profile variability, in classical Be star $V / R$ cycle form. V/R variability increasing in amplitude with period $\sim 550$ days - suspiciously close to the proposed 580 day orbital period. EW decreases slightly, and peak separation reaches minimum, as seen in Phase $1\left(\sim 100 \mathrm{~km} \mathrm{~s}^{-1}\right)$.

He I 6678: Multi-component structure shows strong variability in both intensity and radial velocity, for all peaks. Pairs of peaks converge (Figure 3, right panel), and EW decreases to pre-plateau level (start of Phase 3).

\section{Discussion}

We find a V/R cycle which starts around the time of the optical maximum in $1994 / 95$, with a short period ( $\sim 550$ days). Throughout this $V / R$ cycle we see the disk expanding. The $\mathrm{H} \alpha$ observations show that the disk reforms close to the stellar photosphere (peak separation $\sim 500 \mathrm{~km} \mathrm{~s}^{-1}$ ) reaching a size comparable to that seen in the late 1980's around $\sim 5-6$ years later (peak separation $\sim 180 \mathrm{~km} \mathrm{~s}^{-1}$ ).

The system displays spectroscopic behaviour typical of classical Be stars (Phases 1-3; disk loss and recovery), although the amplitude of the associated photometric variability is unusually large $\left(\Delta V \sim 0.6^{m}\right)$. This period is followed by unusual activity which appears to correspond to complex structural changes in the evolution of the disk. We previously proposed that this might represent a multi-component disk structure (a "double disk"), and this additional data appears to support the interpretation. Observations of the He I6678 line show what could be three separate disc components forming over a period of $\sim 7$ years.

A full analysis of the observations presented here will be published in Roche et al. (in prep.).

Acknowledgments. We thank Drs. L. Kaper, J. Telting and M. Smith for useful discussion and comments on the observations presented here. 